\title{
Trafficking of Plasmodium falciparum chimeric rhoptry protein with Brefeldin A
}

\author{
Ahmed M. Ghoneim \\ Zoology Department, Faculty of Science, Damietta University, New Damietta, Egypt
}

\begin{abstract}
Trafficking of the rhoptry chimeric protein RhopH2GFP, which contains RhopH2 signal peptide plus the downstream five amino acids, was dissected by treating parasites with Brefeldin A at three different time points. Twenty eight hrsstage trophozoites accumulated the chimera within the parasite endoplasmic reticulum. In $32 \mathrm{hrs}$-stage schizonts, the chimera was distributed in the parasite cytoplasm but not in the parasitophorous vacuole. In $36 \mathrm{hrs}$ stage-schizonts, the chimera was detected in individual structures around the developing merozoites and, in contrary to non-treated parasites, no immature rhoptry vesicles could be detected in the cytoplasm of immature merozoites. These data show that this chimera is trafficked to the rhoptries via Brefeldin A-sensitive pathway indicating that this trafficking is similar to that of the endogenous rhoptry proteins, and that the five amino acids downstream of the signal peptide cleavage site may contain the sorting signal required for rhoptry targeting.
\end{abstract}

Keywords: green fluorescent protein, apical secretions, targeting, organelles, merozoite, Apicomplexa

Invasion of erythrocytes by Plasmodium merozoites is a tightly regulated multi-step process. This rapid process begins with the release of proteins from the micronemes that are believed to be involved in the attachment of extracellular parasites to the host membrane, which is then followed by secretion of rhoptry proteins. Secretion of rhoptry proteins is initiated very rapidly after intimate contact of the parasite and the host cell, and is completed within a few minutes of invasion, implying that the contents of rhoptries are vital in the establishment of the parasitophorous vacuole. In comparison to microneme proteins, the role of rhoptry proteins is less well understood in apicomplexan parasites (Dowse and Soldati. 2005, Bradley and Sibley. 2007, Kaneko 2007, Kats et al. 2008, Tufet-Bayona et al. 2009).

Rhoptry proteins are found either in the rhoptry neck or in the rhoptry bulb, and no protein has been observed in both, suggesting that these two locations are discrete compartments (Kawase et al. 2007). In Plasmodium, it was suggested that the rhoptries fuse with the micronemes to facilitate release of their contents (Zhao and Satir1998).

A total of 20 proteins have been identified in the rhoptries of Plasmodium merozoites (Kaneko 2007, Haase et al. 2008,
Wickramarachchi et al. 2008). These include, among others, the high molecular mass RhopH complex, which consists of three proteins: RhopH1, RhopH2, and RhopH3 (Campbell et al. 1984, Holder et al. 1985, Cooper et al. 1988, Hienne et al. 1998). The RhopH complex is conserved across Plasmodium species and seems to be secreted from the rhoptry bulb of mature merozoites. After invasion of erythrocytes, RhopH complex is retained in the newly formed ring stage parasites (Ling et al. 2003). The exact function of the complex has not been determined yet but the complex is thought to be involved in erythrocyte binding during or after invasion (Sam-Yellowe 1992, Rungruang et al. 2005), and perhaps participates in the formation of the parasitophorous vacuolar membrane (Ling et al. 2003, 2004).

Unlike proteins targeted to the apicoplast (Foth et al. 2003) or exported into the host erythrocyte (Hiller et al. 2004, Marti et al. 2004), there is no common targeting signal for rhoptry proteins. The accurate targeting of rhoptry proteins to their final destination is an essential process for the invasion of erythrocytes and the growth of parasites. In a previous study (Ghoneim et al. 2007), we determined that the N-terminal 24 amino acids of RhopH2, including signal peptide sequence, are sufficient to target green fluorescent protein (GFP) to the rhoptries and we proposed that this targeting is likely mediated by a unique mechanism that depends on the interaction with $\mathrm{N}$-terminal 24 amino acids of RhopH2 early in the secretory pathway. In this report, we dissected the trafficking of RhopH2-GFP to the rhoptry organelles under the effect of Brefeldin A.

In the RhopH2-GFP chimera, GFP is expressed under the control of the rhoph 2 promoter together with the sequence encoding RhopH2 signal peptide plus the downstream 5 amino acids (amino acid sequence: MIKVTIFLLLSIFSFNLYG LELNE). This chimera has been previously shown by Ghoneim et al. (2007) to be targeted to the rhoptry efficiently. To dissect the trafficking of this chimera, parasites cultures expressing this chimera were synchronized with 5\% Sorbitol twice after $26 \mathrm{hrs}$ window and divided into four cultures. One was maintained as a control and the other three were treated at three different schizont stages with Brefeldin A (Sigma) at $5 \mu \mathrm{M}$ final concentration. Brefeldin A (BFA) was applied in the new parasite cycle after culture synchronization. Small aliquots of parasite culture were washed and incubated with PBS solution containing the nuclear stain 4', 6-diamidino-2-phenylindole (DAPI) for 


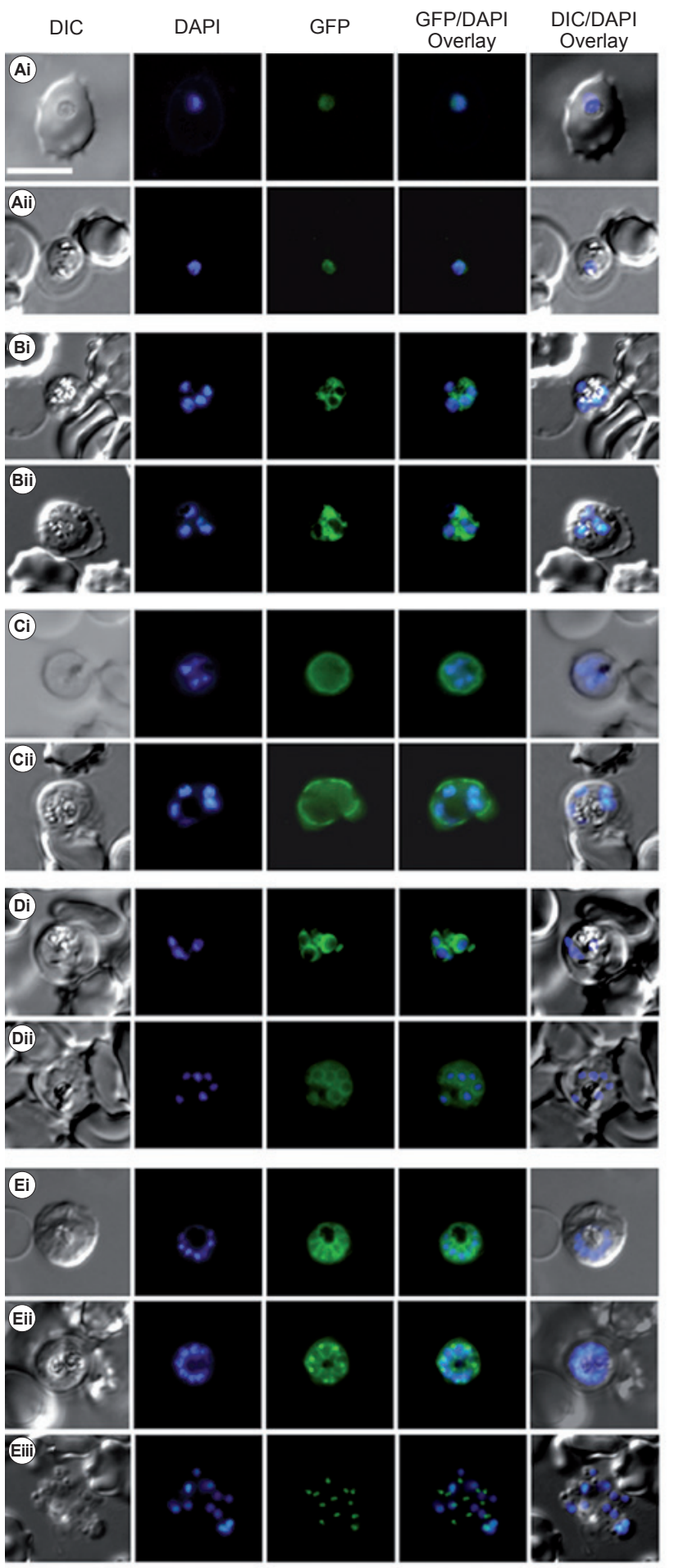

5 min. Parasites were mounted without further treatment and GFP fluorescent parasites were imaged. In the first treatment, 28 hrs-stage trophozoites were treated with BFA and observed regularly for the next 18 hours. The RhopH2-GFP chimeric protein accumulated within these parasites in a structure that surrounds the parasite nucleus (Fig. 1, panels Ai and Aii). This fluorescence pattern is consistent with distribution of the chimera in the whole endoplasmic reticulum. In the second treatment, schizonts were treated $32 \mathrm{hrs}$ post invasion with BFA and observed regularly for the next 14 hours. As in Fig. 1 (panels Bi adn B-ii), the RhopH2-GFP chimeric protein accumulated in the whole parasite cytoplasm and no green fluorescence signal was detected at the periphery of the parasite. In the nontreated parasites, however, the RhopH2-GFP chimeric protein was detected in the whole parasite cytoplasm and accumulated in the parasitophorous vacuole (Fig. 1, panels $\mathrm{Ci}$ and $\mathrm{Cii}$ ). This indicates that BFA blocks trafficking of RhopH2-GFP chimeric protein to the parasitophorous vacuole.

In the third treatment, $36 \mathrm{hrs}$ stage-schizonts were treated with BFA and monitored for the next 10 hours. As in Fig. 1 (panels Di and Dii), the RhopH2-GFP chimeric protein notably accumulated in individual structures around each newly formed merozoite. No green fluorescent vesicles were seen in the cytoplasm of any immature merozoite. In the non-treated parasites, moderate and obviously lower amounts of RhopH2-GFP chimeric protein were detected in individual structures around the developing merozoites. In addition, clearly fluorescent vesicles that might represent immature rhoptry organelles could be seen within the cytoplasm of the newly formed merozoites (Fig. 1, panels Ei and Eii). Moreover, all BFA treated-parasite cultures never showed segmented schizonts with apically fluorescent merozoites while the non-treated parasites matured and released merozoites with apically fluorescent rhoptry organelles (Fig. 1, panel Eiii). Altogether, these data indicate that the targeting of the chimeric protein RhopH2-GFP is BFA sensitive and that this protein is trapped around individual merozoites under the effect of BFA.

This finding coincides with previous finding that targeting to rhoptry organelle is BFA-sensitive (Howard and Schmidt 1995, Noe et al. 2000). This also implies that targeting of the chimera RhopH2-GFP is similar to that of the endogenous rhoptry proteins, at least at the early secretory pathway (ER/Golgi). BFA inhibits protein secretion and has been used to investigate the secretory pathway (Klausner et al. 1992). It binds the sec7 domain of some ADP-Ribosylation Factor-Guanine nucleotide Exchange Factors (ARF-GEFs) and disrupts interaction with their ARF partners (Mossessova et al. 2003), which are essential for vesicle formation at the ER-Golgi interface. Therefore, their inhibition stops anterograde transport from the ER to Golgi (Tonkin et al. 2006). In the present study, the BFA-induced inhi-

Fig. 1. Treatment of the parasite line expressing the chimeric protein RhopH2-GFP at different stages with $5 \mu \mathrm{M}$ Brefeldin A. A - late trophozoite stages ( $28 \mathrm{hrs)}$ treated with Brefeldin A. The chimera seems to be distributed in the whole endoplasmic reticulum. B - Early stage schizonts ( $\sim 32 \mathrm{hrs})$ treated with Brefeldin A. The chimeric protein was distributed in the whole parasite cytoplasm and did not reach the parasitophorous vacuole. $\mathbf{C}$ - Non-treated early stage schizonts with the chimeric protein distributed in both the parasite cytoplasm and the parasitophorous vacuole. D - Middle stage schizonts ( $\sim 36 \mathrm{hrs})$ treated with Brefeldin A. The chimera notably accumulated in individual structures around each newly formed merozoite and vesicles representing immature rhoptries could not be detected in the cytoplasm of any immature merozoite. $\mathbf{E}$ - Non-treated parasites grew, matured and released apically punctated merozoites. The RhopH2-GFP chimera was detected in a segmented pattern around the developing merozoites and clearly fluorescent vesicles representing immature rhoptry organelles could be also seen within the cytoplasm of the newly formed merozoites. Scale bar $=5 \mu \mathrm{m}$. 
bition of RhopH2-GFP targeting to the parasitophorous vacuole implies that this protein moves from the ER-Golgi to the parasitophorous vacuole. Whether this targeting to the parasitophorous vacuole is a default destination before the formation of rhoptry organelles remains to be unveiled.

Cleavage of the signal peptide of the RhopH2-GFP at the ER leaves only five amino acids from the RhopH2 fused to GFP. We have previously suggested that this chimeric protein is likely to be recognized by a chaperone, for example Hsp70 family or its homologues, in the ER matrix through interaction with the first 24 amino acid sequence of the RhopH2. A simultaneous binding to the mature protein by a specific co-chaperone or co-factor would then mark these proteins for binding with a rhoptry targeted molecule (Ghoneim et al. 2007). It is possible that the signal peptide itself encodes some targeting information. However, Tonkin et al. (2006) demonstrated that, for the apicoplast targeted protein ACP, the function of signal peptide is to recruit proteins, regardless of their structure, to general secretory pathway. In general, protein recognition often involves extensive protein-protein interactions but there are some cases in which short amino acid sequences are recognized.

Recently, several studies demonstrated that the few amino acids next to the signal peptide cleavage site contain or represent specific targeting information. According to Eksi and Williamson (2011), a GFP chimeric protein consisting of the signal peptide sequence plus the next five amino acids of the Pfs 16 and expressed under its promoter was sufficiently and correctly localized to the gametocyte surface. Replacement of the RAP1 signal sequence with a signal sequence from ACP (as an example for a non-rhoptry protein) had no effect on rhoptry localization indicating that the function of signal sequence of RAP1 is to direct the protein into the secretory pathway.

Information contained in amino acids 22-55 seems to represent a RAP1 rhoptry signal that is necessary and sufficient for optimal targeting to the rhoptries. This RAP1 rhoptry signal interacts with the C-terminus of RAMA (Richard et al. 2009), thus providing evidence that RAMA, GPI-anchored protein in the Golgi lumen, acts as an escorter for the low molecular weight complex. Mutation analysis of specific residues after the signal peptide indicated that although residues Y47 and W48 play a significant role in RAP1 targeting, it is the overall nature of the RAP1 rhoptry signal that is important. The RAP1 rhoptry signal is specific for the low molecular weight complex because RAP1 orthologues from dif- ferent Plasmodium spp. did not identify any potential conserved motifs within the RAP1 rhoptry signal (Richard et al. 2009). According to Osborne et al. (2010), the HT motif (RxLxE/D/Q) of exported proteins, also referred to as a Plasmodium export element (PEXEL), is cleaved and N-acetylated in the parasite ER. Recognition of a protein with a cleaved HT motif would likely involve recognition of only a few residues including the acetylated $\mathrm{N}$-terminal residue and the $\mathrm{E} / \mathrm{D} / \mathrm{Q}$ residue in the fifth position of the motif (Osborne et al. 2010).

Gaji et al. (2011) showed that valine at position +3 (relative to signal peptidase cleavage site) of the propeptide of TgM2AP and leucine at position +1 of the propeptide of TgMIC 5 in these two micronemal proteins are crucial for targeting to micronemes. Substitution of valine by leucine and vice versa did not affect correct trafficking of the mutated proteins. Interestingly, propeptides of TgAMA1, TgMIC3 and Eimeria tenella MIC5 have valine or leucine at or near the $\mathrm{N}$-termini and mutagenesis of these conserved residues validated their role in microneme trafficking. Collectively, these findings suggested that discrete, aliphatic residues at the extreme N-termini of propeptides of the apicomplexan microneme proteins facilitate trafficking to the micronemes (Gaji et al. 2011). Deletion of the first two amino acids of the TgMIC5 propeptide impaired trafficking to the micronemes (Gaji et al. 2011). Interestingly, our chimeric protein RhopH2-GFP contains two leucine residues downstream of the signal peptide cleavage site and it is highly possible that these two leucine residues play a role in the trafficking of this chimera.

Altogether, these data provide an evidence for the importance of the first few amino acids downstream of the signal peptide cleavage site in the trafficking of soluble rhoptry proteins. Regardless of the exact mechanism by which such proteins could be targeted, it is likely that these few amino acids are recognized in the ER by specific molecules that would define a targeting pathway. Identification of specific amino acid residues involved in the trafficking of proteins to the rhoptry may lead to the development of small molecules able to interfere with the biogenesis of this vital compartment and the cell invasion process, which might be ultimately used as drugs for the treatment of malaria.

Acknowledgements. I would like to thank Prof. A. Khidr, Zoology Department, Faculty of Science, Damietta University, for his help and critical reading of the manuscript.

\section{References}

Bradley P.J., Sibley L.D. 2007: Rhoptries: an arsenal of secreted virulence factors. Curr. Opin. Microbiol. 10: 582-587.

Campbell G.H., Miller L.H., Hudson D., Franco E.L., AnDRYSIAK P.M. 1984: Monoclonal antibody characterization of Plasmodium falciparum antigens. Am. J. Trop. Med. Hyg. 33: 1051-1054.

Cooper J.A., Ingram L.T., Bushell G.R., Fardoulys C.A., Stenzel D., Schofield L., Saul A.J. 1988: The 140/130/105 kilodalton protein complex in the rhoptries of Plasmodium falciparum consists of discrete polypeptides. Mol. Biochem. Parasitol. 29: 251-260.

Dowse T.J., Soldati D. 2005: Rhomboid-like proteins in Apicomplexa: phylogeny and nomenclature. Trends Parasitol. 21: 254-258.

Eksi S., Williamson K.C. 2011: Protein targeting to the parasitophorous vacuole membrane of Plasmodium falciparum. Eukaryot. Cell 10: 744-752.

Foth B.J., Ralph S.A., Tonkin C.J., Struck N.S., Fraunholz M., Roos D.S., Cowman A.F., McFadden G.I. 2003: Dissecting apicoplast targeting in the malaria parasite Plasmodium falciparum. Science 299: 705-708.

Gaji R.Y., Flammer H.B., Carruthers V.B. 2011: Forward targeting of Toxoplasma gondii proproteins to the micronemes involves conserved aliphatic amino acids. Traffic 12: 840-853.

Ghoneim A., Kaneko O., Tsuboi T., Toril M. 2007: The Plasmodium falciparum $\mathrm{RhopH} 2$ promoter and first 24 amino acids are sufficient to target proteins to the rhoptries. Parasitol. Int. 56: $31-43$. 
Haase S., Cabrera A., Langer C., Treeck M., Struck N., Herrmann S., Jansen P.W., Bruchhaus I., Bachmann A., Dias S., Cowman A.F., Stunnenberg H.G., Spielmann T., GilBERGER T.W. 2008: Characterization of a conserved rhoptry associated leucine zipper-like protein in the malaria parasite Plasmodium falciparum. Infect. Immun. 76: 879-887.

Hienne R., Ricard G., Fusaï T., Fujioka H., Pradines B., Aikawa M., Doury J.C. 1998: Plasmodium yoelii: identification of rhoptry proteins using monoclonal antibodies. Exp. Parasitol. 90: 230-235.

Hiller N.L., Bhattacharjee S., van Ooij C., Liolios K., Harrison T., Lopez-Estraño C., Haldar K. 2004: A host-targeting signal in virulence proteins reveals a secretome in malarial infection. Science 306: 1934-1937.

Holder A.A., Freeman R.R., Uni S., Aikawa M. 1985: Isolation of a Plasmodium falciparum rhoptry protein. Mol. Biochem. Parasitol. 14: 293-303.

Howard R.F., Schmidt C.M. 1995: The secretory pathway of Plasmodium falciparum regulates transport of p82/RAP1 to the rhoptries. Mol. Biochem. Parasitol. 74: 43-54.

KANEKO O. 2007: Erythrocyte invasion: vocabulary and grammar of the Plasmodium rhoptry. Parasitol. Int. 56: 255-262.

Kats L.M., Cooke B.M., Coppel R.L., Black C.G. 2008: Protein trafficking to apical organelles of malaria parasites - building an invasion machine. Traffic 9: 176-186.

Kawase O., Nishikawa Y., Bannai H., Zhang H., Zhang G., Jin S., Lee E.G., Xuan X. 2007: Proteomic analysis of calcium-dependent secretion in Toxoplasma gondii. Proteomics 7: $3718-3725$.

Klausner R.D., Donaldson J.G., Lippincott-Schwartz J. 1992: Brefeldin A: insights into the control of membrane traffic and organelle structure. J. Cell Biol. 116: 1071-1080.

Ling I.T., Florens L., Dluzewski A.R., Kaneko O., Grainger M., Yim Lim B.Y. Tsuboi T., Hopkins J.M., Johnson J.R., Toril M., Bannister L.H., Yates J.R. III, Holder A.A., Mattei D. 2004: The Plasmodium falciparum clag9 gene encodes a rhoptry protein that is transferred to the host erythrocyte upon invasion. Mol. Microbiol. 52: 107-118.

Ling I.T., Kaneko O., Narum D.L., Tsuboi T., Howell S., Taylor H.M., Scott-Finnigan T.J., Tori M., Holder A.A. 2003: Characterisation of the rhoph2 gene of Plasmodium falciparum and Plasmodium yoelii. Mol. Biochem. Parasitol. 127: 47-57.

Marti M., Good R.T., Rug M., Knuepfer E., Cowman A.F. 2004: Targeting malaria virulence and remodeling proteins to the host erythrocyte. Science 306: 1930-1933.
Mossessova E., Corpina R.A., Goldberg J. 2003: Crystal structure of ARF1*Sec7 complexed with Brefeldin A and its implications for the guanine nucleotide exchange mechanism. Mol. Cell. 12: 1403-1411.

Noe A.R., Fishrind D.J., Adams J.H. 2000: Spatial and temporal dynamics of the secretory pathway during differentiation of the Plasmodium yoelii schizont. Mol. Biochem. Parasitol. 108: $169-185$.

Osborne A.R., Speicher K.D., Tamez P.A., Bhattacharjee S., Speicher D.W., Haldar K. 2010: The host targeting motif in exported Plasmodium proteins is cleaved in the parasite endoplasmic reticulum. Mol. Biochem. Parasitol. 171: 25-31.

Richard D., Kats L.M., Langer C., Black C.G., Mitri K., Boddey J.A., Cowman A.F., Coppel R.L. 2009: Identification of rhoptry trafficking determinants and evidence for a novel sorting mechanism in the malaria parasite Plasmodium falciparum. PLoS Pathog. 5 (3): e1000328.

Rungruang T., Kaneko O., Murakami Y., Tsuboi T., Hamamoto H., Akimitsu N., Sekimizu K., Kinoshita T., Tori M. 2005: Erythrocyte surface glycosylphosphatidyl inositol anchored receptor for the malaria parasite. Mol. Biochem. Parasitol. 140: 13-21.

Sam-Yellowe T.Y. 1992: Molecular factors responsible for host cell recognition and invasion in Plasmodium falciparum. J. Protozool. 39: 181-189.

Tonkin C.J., Struck N.S., Mullin K.A., Stimmler L.M., McFADDEN G.I. 2006: Evidence for Golgi-independent transport from the early secretory pathway to the plastid in malaria parasites. Mol. Microbiol. 61: 614-630.

Tufet-Bayona M., Janse C.J., Khan S.M., Waters A.P., Sinden R.E., Franke-Fayard B. 2009: Localisation and timing of expression of putative Plasmodium berghei rhoptry proteins in merozoites and sporozoites. Mol. Biochem. Parasitol. 166: 22-31.

Wickramarachchi T., Devi Y.S., Mohmmed A., Chauhan V.S. 2008: Identification and characterization of a novel Plasmodium falciparum merozoite apical protein involved in erythrocyte binding and invasion. PLoS ONE 3: e1732.

Zhao H., Satir B.H. 1998: Parafusin is a membrane and vesicle associated protein that cycles at exocytosis. Eur. J. Cell Biol. 75: $46-53$

Received 5 July 2012

Accepted 3 October 2012 